\title{
$T=\left(\frac{\partial S}{\partial U}\right)^{-1} ?$
}
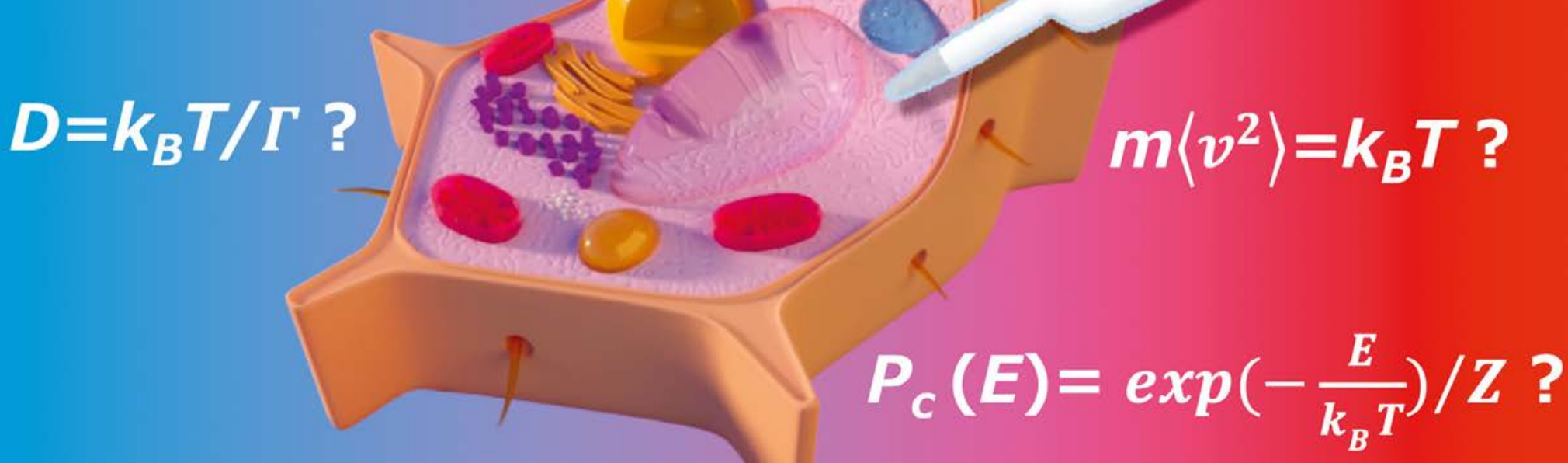

\section{WHAT IS THE TEMPERATURE OF
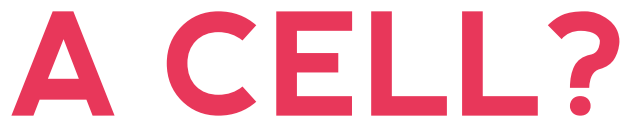

\footnotetext{
- Kumiko Hayashi, ${ }^{1,2}$, Shin Hasegawa1 and Shinsuke Niwa ${ }^{3}$ - Dol: https://doi.org/10.1051/epn/2020510

- ${ }^{1}$ Department of Applied Physics, Graduate School of Engineering, Tohoku University, Sendai, Japan

- ${ }^{2}$ Precursory Research for Embryonic Science and Technology (PRESTO), Japan Science and Technology Agency (JST), Tokyo, Japan

- ${ }^{3}$ Frontier Research Institute for Interdisciplinary Sciences and Graduate School of Life Science, Tohoku University, Sendai, Japan

ICorrespondence: kumiko@camp.apph.tohoku.ac.jp
}

\section{Equilibrium temperature is classically defined based on thermodynamics using entropy and internal energy: how then can we describe temperature in non-equilibrium living systems such as cells, whose states are not well described by existing thermodynamics?}

rom the viewpoint of physics, temperature $(T)$ is defined from several theoretical standpoints. With respect to thermodynamics, temperature is defined as the inverse of a partial derivative of entropy $(S)$ with respect to internal energy $(U)$ of a system $(\partial S / \partial U)$. Reaching back to statistical mechanics, temperature then follows the canonical distribution as $p_{c}(E)=\exp \left(-\frac{E}{k_{\mathrm{B}} T}\right) / Z$, where $E$ is energy of a state, $k_{B}$ is the Boltzmann constant, and $Z$ is the partition function. Application of the canonical distribution to a Hamiltonian particle system connected to an equilibrium thermostat then provides the equipartition law regarding the energy of motion: $m\left\langle v^{2}\right\rangle / 2=k_{\mathrm{B}} T / 2$. Further, temperature is included in the fluctuation-dissipation theorem such as the Einstein relation and the Green-Kubo relation, in which the temperature of a system is a proportionality constant between the correlation and response functions.
Thus, the temperature of equilibrium systems is perfectly defined by many physical laws. However, the definition is less straightforward when considering a non-equilibrium system, including living organisms.

\section{Effective temperature under non-equilibrium conditions}

A non-equilibrium state is the state of a system with energy injection, such as driving forces, thermal conductions, and chemical reactions. Thus, almost all systems we are exposed to are non-equilibrium systems, since living matter such as the cells constituting our bodies require continuous energy input, and are thus in non-equilibrium states. Given the much greater complexity of living matter compared to an ideal gas, the statistical properties cannot be well described by the existing thermodynamics and statistical mechanics theories described above. This 
poses a challenge in defining the temperature of such systems that are so far from the equilibrium in which thermodynamics, statistical mechanics, and linear response theories are valid.

In the case of a simple driven system (i.e., a non-equilibrium system), in which a Brownian particle in a periodic potential in a medium at $\mathrm{T}$ is driven by an external force, it was proved that the Einstein relation,

$$
D=k_{B} T / \Gamma,
$$

where $D$ is the diffusion coefficient, and $\Gamma$ is the friction coefficient of the particle), was violated based on theoretically calculating the transport coefficients $D$ (diffusion coefficient) and $\Gamma$ (friction coefficient) of the particle [1]. Then, $T_{\text {eff }}=D / k_{\mathrm{B}} \Gamma$ is called the effective temperature of the non-equilibrium system because $T_{\text {eff }}$ has a dimension of temperature though $T_{\text {eff }} \neq T$.

\section{Intracellular cargo transport}

Indeed, the Einstein relation (Eq. 1) is violated in the real world when considering the state of living matter. Cells contain driven particle systems such as the above-mentioned driven Brownian particle, which is referred to as intracellular cargo transport, in which cargo particles are driven by motor proteins (Fig. 1). Motor proteins generate the transport force by a chemical reaction: hydrolysis of adenosine triphosphate (ATP) molecules. The violation of the Einstein relation (Eq. 1) was clarified experimentally in the intracellular cargo transport by measuring the diffusion coefficient $(D)$ and frictional coefficient $(I)$ of cargos [2].

In eukaryotic cells, cargos-which pack biomaterials required for sustaining life activities-are transported by the motor proteins kinesin and dynein along microtubules acting as the "roads" for transport spreading across a cell (Fig. 1) [3]. In particular, neurons, which have long axons (ranging from $0.1 \mathrm{~mm}$ to $1 \mathrm{~m}$ ) to connect a cell body to the synaptic region, require fast cargo transport (Fig. 2a) [3]. In addition to neuronal communication, intracellular cargo transport is used by fish to change the colour of their body surfaces (Fig. 2b). Melanin pigments aggregate as cargos in cells on the scale of fish by motor proteins to drive a colour change from dark to light depending on environmental cues. Thus, intracellular cargo transport by motor proteins is a significant function of eukaryotic cells.

Because intracellular cargos are typically several hundred nanometres in size, they can be observed by optical microscopes with a spatial resolution of approximately $200 \mathrm{~nm}$ (Fig. 2a,b). Fluorescent proteins and dyes are typically used to label cargos so that they can be readily distinguished in the intracellular environment that is crowded with other vesicles, proteins, and cytoskeletons (Fig. 2a, bottom). By tracking the centre position of a cargo, its trajectory can be obtained (Fig. 2b, bottom). A cargo transported by motor proteins can be seen over several seconds moving at a constant speed $(v)$, while fluctuating due to the effective noise derived from collisions with other vesicles and cytoskeleton components as well as thermal noise in the intracellular environment. This effective noise is considered to be an origin of the effective temperature.

The existence of the effective noise arising in the intracellular non-equilibrium environment causes a problem with respect to physical measurement such as force measurement. The driving force $(F)$ acting on cargo transport exerted by motor proteins, can be written as

$$
F=\Gamma v,
$$

where $\Gamma$ is the friction coefficient of the cargo, however, the value of the friction coefficient $(\Gamma)$ cannot be estimated from the diffusion coefficient $(D)$, which is easily obtained from the trajectory of a cargo (Fig. 2b, bottom), because of the violation of the Einstein relation (i.e., $\Gamma \neq k_{\mathrm{B}} T / D$ ). Therefore, the measurable index $\chi \equiv v / D$ was recently proposed as a substitute for $F$. Using the relation of the effective temperature, the relationship between $F$ and $\chi$ is derived as $F=k_{\mathrm{B}} T_{\text {eff }} \cdot \chi[4,5]$. In Fig. $2 \mathrm{c}, \mathrm{d}, \chi$ for neuronal cargos $(\mathrm{n}=40)$ (Fig. $2 \mathrm{c}$ ) and melanin pigment cargos $(\mathrm{n}=62)($ Fig. $2 \mathrm{~d})$ is plotted as a function of $\Delta \mathrm{t}$ [note that $\Delta \mathrm{t}$ dependence comes from $\left.\mathrm{D}\left(=\left\langle\Delta X^{2}\right\rangle / 2 \Delta \mathrm{t}\right)\right]$ $[4,5]$. The index $\chi$ was found to be discrete, representing the number of motor proteins carrying a single cargo together and generating a driving force. Accordingly, a cargo is transported by multiple motor proteins, similar to the way that many ants work collectively (Fig. 2e).

Using the known force value (approximately $5 \mathrm{pN}$ ) of a single motor protein and the relationship $\mathrm{F}=k_{\mathrm{B}} T_{\text {eff }} \cdot \chi, T_{\text {eff }}$ was estimated to be approximately $10 k_{\mathrm{B}} T$ for neuronal cargo transport [4] and $2 k_{\mathrm{B}} T$ for melanin transport in pigment cells [5]. The reason for this large difference in $T_{\text {eff }}$ between different cell types has not yet been clarified; however, variations in noise properties and cell structures may affect the value of $T_{\text {eff. }}$ Taking into consideration that the amount of energy provided by the hydrolysis of

vFIG. 1:

Schematic of intracellular cargo transport. A motor protein (red) generates driving force $(F)$ to carry a cargo (green) moving at velocity ( $v$ ) along a microtubule.

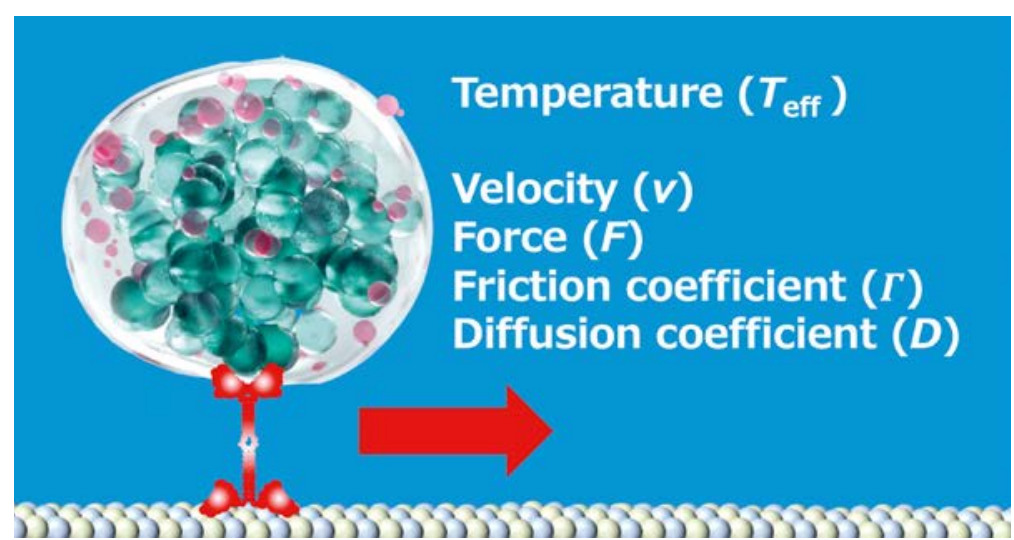


a
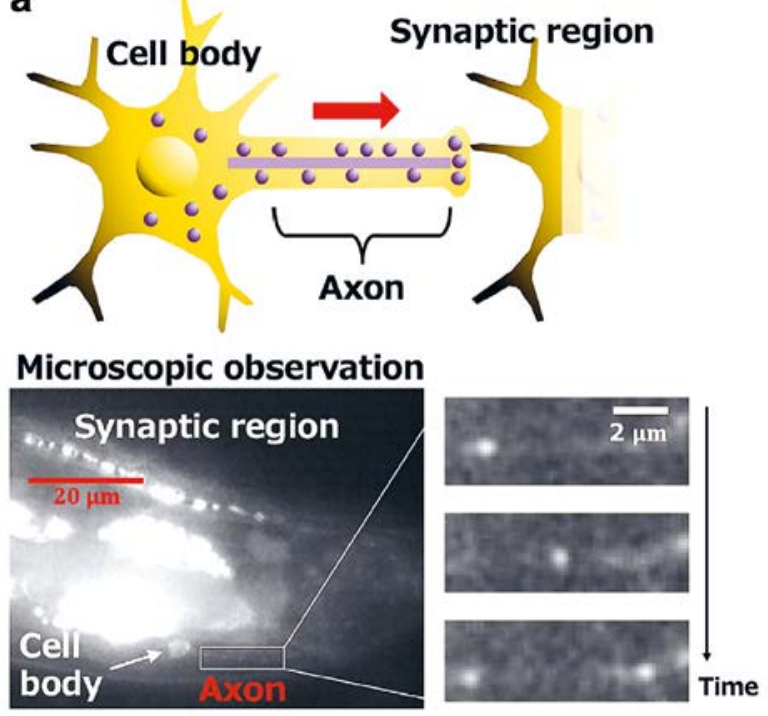

b

- FIG. 2:

(a) Synaptic vesicle precursor transport in a neuron [4]. (b) Melanin pigment transport in a pigment cell [5]. Measurement of the force index $\chi$ in the case of neuronal cargo transport [4] (c) and melanin pigment transport [5] (d) (e) A single cargo is carried by multiple motor proteins, akin to ant-like transport.
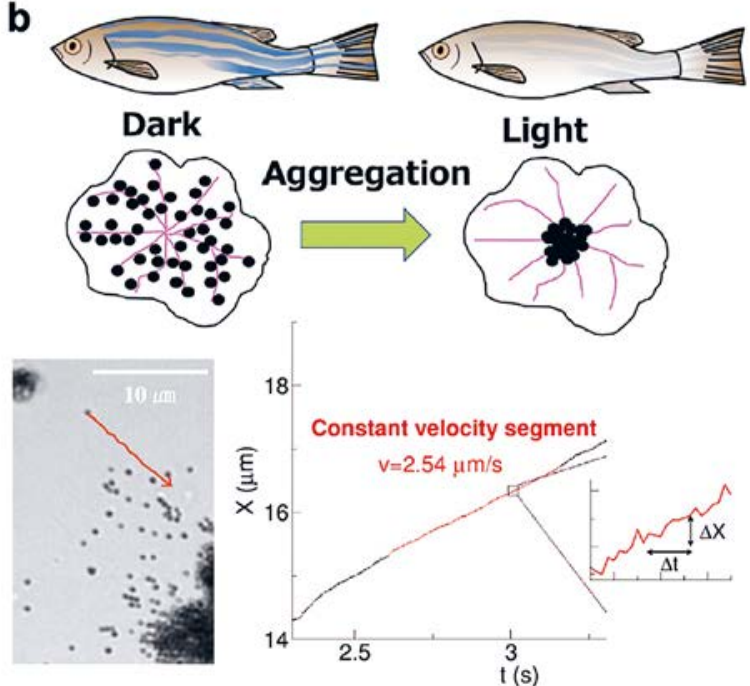

one ATP molecule is also of the order of $10 k_{\mathrm{B}} T$, the energetic meaning of $T_{\text {eff }}$ is an important issue to be solved for realising force measurement of the intracellular environment.

\section{Application of $X$}

Although the exact value of the proportionality constant $k_{\mathrm{B}} T_{\text {eff }}$ between $F$ and $\chi$ is unknown [note that $\left.F=k_{\mathrm{B}} T_{\text {eff }} \cdot \chi\right]$, the qualitative property of $\chi$, namely its discreteness (Fig. 2c,d), is significant, as the discreteness implies the number of motor proteins carrying a cargo, which represents the stability of material transport and can therefore serve as a good barometer of healthy transport. Indeed, a decrease in the number of motor proteins observed in synaptic cargo transport was found to result in the mislocation of synapses $[4,6]$. In neurons, deficits in axonal transport are strongly related to neuronal diseases, including Alzheimer's, Parkinson's, and Huntington's diseases. Therefore, the measurement of $\chi$ has potential to be more widely applied in the field of neuroscience as a helpful index to monitor disease progression and severity.

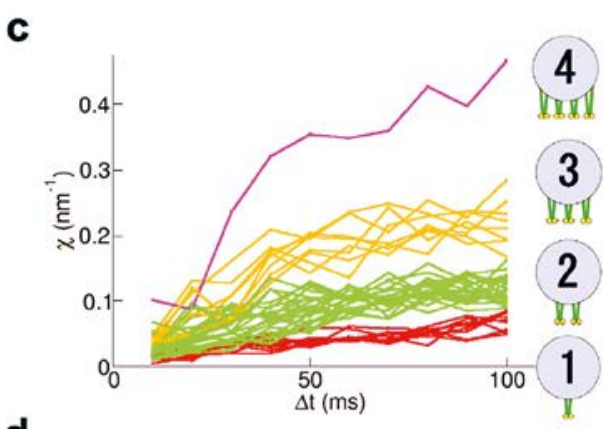

d

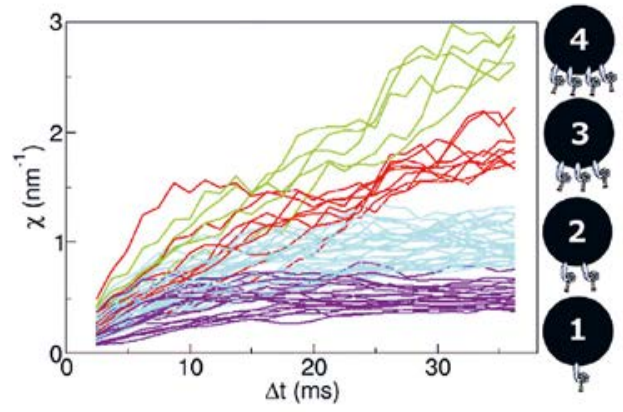

e

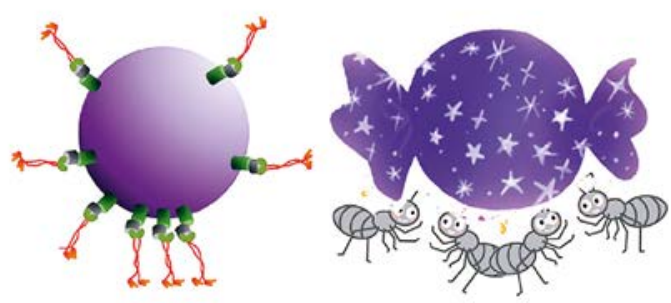

About the Authors

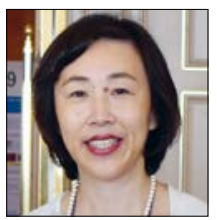

Kumiko Hayashi is an associate professor of the Applied Physics Department, Tohoku University in Japan. She changed her major from theoretical physics to biological experiments after acquiring a $\mathrm{PhD}$. Currently, she works on both the fields of theory and experiment fields. Shin Hasegawa graduated from the Applied Physics Department, Tohoku University in Japan. He got a master's degree in engineering.

Shinsuke Niwa is an associate professor of the Frontier Research Institute for Interdisciplinary Sciences (FRIS), Tohoku University in Japan.

\section{References}

[1] K. Hayashi and S. Sasa, Phys. Rev. E69, 066119 (2004)

[2] K. Hayashi, et al., Eur. Phys. J. E 36, 136 (2013)

[3] N. Hirokawa, et al., Nat. Rev. Mol. Cell. Biol. 10, 682 (2009)

[4] K. Hayashi, et al., Phys. Chem. Chem. Phys. 20, 3403 (2018)

[5] S. Hasegawa, et al., Sci. Rep. 9, 5099 (2019)

[6] S. Niwa, et al., Cell Reports 16, 1 (2016) 